\title{
The Effect of Variations in Ionic Conductance Values on the Suppression of Repetitive Spiking in a Mathematical Model of Type-A Medial Vestibular Nucleus Neurons
}

\author{
Takaaki Shirahata \\ Institute of Neuroscience and Kagawa School of Pharmaceutical Sciences, Tokushima Bunri University, Sanuki, \\ Japan \\ Email: tshi@kph.bunri-u.ac.jp
}

Received 22 April 2016; accepted 21 June 2016; published 24 June 2016

Copyright (C) 2016 by author and Scientific Research Publishing Inc.

This work is licensed under the Creative Commons Attribution International License (CC BY).

http://creativecommons.org/licenses/by/4.0/

(c) (i) Open Access

\section{Abstract}

A previous study has proposed a mathematical model of type-A medial vestibular nucleus neurons (mVNn). This model is described by a system of nonlinear ordinary differential equations, which is based on the Hodgkin-Huxley formalism. The type-A mVNn model contains several ionic conductances, such as the sodium conductance, calcium conductance, delayed-rectifier potassium conductance, transient potassium conductance, and calcium-dependent potassium conductance. The previous study revealed that spontaneous repetitive spiking in the type-A mVNn model can be suppressed by hyperpolarizing stimulation. However, how this suppression is affected by the ionic conductances has not been clarified in the previous study. The present study performed numerical simulation analysis of the type-A mVNn model to clarify how variations in the different ionic conductance values affect the suppression of repetitive spiking. The present study revealed that the threshold for the transition from a repetitive spiking state to a quiescent state is differentially sensitive to variations in the ionic conductances among the different types of ionic conductance.

\section{Keywords}

Mathematical Model, Numerical Simulation, Type-A Medial Vestibular Nucleus Neurons, Ionic Conductance

\section{Introduction}

Type-A medial vestibular nucleus neurons (mVNn) can show spontaneous repetitive spiking without stimulation

How to cite this paper: Shirahata, T. (2016) The Effect of Variations in lonic Conductance Values on the Suppression of Repetitive Spiking in a Mathematical Model of Type-A Medial Vestibular Nucleus Neurons. Applied Mathematics, 7, 11341139. http://dx.doi.org/10.4236/am.2016.710101 
in vitro. A mathematical model, which reproduces the spiking, has been proposed based on the Hodgkin-Huxley formulation [1]. This model, which is described by a system of nonlinear ordinary differential equations (ODEs), contains several ionic conductances such as the sodium conductance, calcium conductance, delayed-rectifier potassium conductance, transient potassium conductance, and calcium-dependent potassium conductance. The study that proposed this model also indicated that the spontaneous spiking activity of the type-A mVNn model can be suppressed by hyperpolarizing stimulation [1]. However, it is important to conduct detailed analysis of the ionic conductances of excitable cells [2]. Thus, it is important to clarify how the different ionic conductances in the type-A mVNn model each affect the suppression of spiking. However, this issue was not been investigated in the previous study [1].

Several studies have investigated the characteristics of the ionic conductances of various excitable cell models. For example, various studies have investigated the effect of co-variation of ionic conductances on the dynamics of excitable cell models [3]-[12]. In addition, studies have investigated the effect of variation of ionic conductances on the electrical stimulation-induced changes in the dynamical states [13]-[16]. The above studies have contributed to an understanding of the differences between the characteristics among different ionic conductances. Although analysis of ionic conductances has been extensively performed as described above, analysis of the ionic conductances of the type-A mVNn model has not been performed. Investigation of the type-A mVNn model will contribute to an understanding of the difference in the characteristics among ionic conductances of the type-A mVNn model in more detail. Therefore, the present study performed numerical simulation of the type-A mVNn model to clarify how the suppression of the spiking is dependent on the different ionic conductances.

\section{Materials and Methods}

The present study performed numerical simulations of a mathematical model of type-A mVNn, which was developed in a previous study [1]. The model is described by a system of nonlinear ODEs, which consists of the following five state variables: the membrane potential of the $\mathrm{mVNn}[\mathrm{V}(\mathrm{mV})]$, three gating variables of ionic currents $(n, x$, and $b)$, and calcium concentration $\{[C a](\mu \mathrm{M})\}$. The time evolution of the state variables is described as follows:

$$
\begin{aligned}
& C_{m} \frac{\mathrm{d} V}{\mathrm{~d} t}=I_{a p p}-I_{N a}(V, n)-I_{C a}(V, x,[C a]) \\
& -I_{K}(V, n)-I_{K C a}(V,[C a])-I_{A}(V, b)-I_{L}(V) \\
& \frac{\mathrm{d} n}{\mathrm{~d} t}=\frac{1}{\tau_{n}(V)}\left(n_{\infty}(V)-n\right) \\
& \frac{\mathrm{d} x}{\mathrm{~d} t}=\frac{1}{\tau_{x}(V)}\left(x_{\infty}(V)-x\right) \\
& \frac{\mathrm{d} b}{\mathrm{~d} t}=\frac{1}{\tau_{b}(V)}\left(b_{\infty}(V)-b\right) \\
& \frac{\mathrm{d}[C a]}{\mathrm{d} t}=-K_{P} I_{C a}(V, x,[C a])-R[C a]
\end{aligned}
$$

where $C_{m}\left(1 \mu \mathrm{F} / \mathrm{cm}^{2}\right)$ is the membrane capacitance; $I_{a p p}$ is the externally injected current of constant amplitude; $I_{N a}(V, n), I_{C a}(V, x,[C a]), I_{K}(V, n), I_{K C a}(V,[C a]), I_{A}(V, b)$, and $I_{L}(V)$ are the sodium current, calcium current, delayed-rectifier potassium current, calcium-dependent potassium current, transient potassium current, and leak current, respectively, which are defined in Equations (6)-(11) below; $\tau_{X}(V)$ (ms) $(X=n, x, b)$ and $X_{\infty}(V)(X=n, x$, $b)$ are the time constants of activation/inactivation and the steady-state activation/inactivation functions, respectively, which are defined in Equations (12)-(17) below; $K_{p}(=1)$ is the calcium influx parameter; and $R(=5)$ is the parameter that regulates calcium removal through calcium transport and mitochondrial uptake.

$$
I_{N a}(V, n)=g_{N a}\left(\frac{1}{1+\mathrm{e}^{-0.11(V+33)}}\right)^{3}(1-n)\left(V-V_{N a}\right)
$$




$$
\begin{aligned}
& I_{C a}(V, x,[C a])=g_{C a} x^{2}\left(\frac{1}{1+[C a]}\right)\left(V-V_{C a}\right) \\
& I_{K}(V, n)=g_{K} n^{4}\left(V-V_{K}\right) \\
& I_{K C a}(V,[C a])=g_{K C a}\left(\frac{[C a]}{0.5+[C a]}\right)\left(V-V_{K}\right) \\
& I_{A}(V, b)=g_{A}\left(\frac{1}{1+\mathrm{e}^{-0.1(V+40)}}\right) b\left(V-V_{K}\right) \\
& I_{L}(V)=g_{L}\left(V-V_{L}\right) \\
& \tau_{n}(V)=\frac{1}{0.1 \mathrm{e}^{0.055(V+40)}+0.1 \mathrm{e}^{-0.055(V+40)}} \\
& \tau_{x}(V)=5 \\
& \tau_{b}(V)=10 \\
& n_{\infty}(V)=\frac{1}{1+\mathrm{e}^{-0.1((V+40)}} \\
& x_{\infty}(V)=\frac{1}{1+\mathrm{e}^{-0.16(V+30)}} \\
& b_{\infty}(V)=\frac{1}{1+\mathrm{e}^{0.2(V+70)}}
\end{aligned}
$$

where $g_{N a}, g_{C a}, g_{K}, g_{K C a}, g_{A}$, and $g_{L}\left(=0.3 \mathrm{mS} / \mathrm{cm}^{2}\right)$ are the maximal conductances of $I_{N a}(V, n), I_{C a}(V, x,[C a])$, $I_{K}(V, n), I_{K C a}(V,[C a]), I_{A}(V, b)$, and $I_{L}(V)$, respectively, and $V_{N a}(=55 \mathrm{mV}), V_{C a}(=124 \mathrm{mV}), V_{K}(=-80 \mathrm{mV})$, and $V_{L}(=-50 \mathrm{mV})$ are the reversal potentials of $I_{N a}(V, n), I_{C a}(V, x,[C a])$, three types of potassium currents $\left\{I_{K}(V, n)\right.$, $I_{K C a}(V,[C a])$, and $\left.I_{A}(V, b)\right\}$, and $I_{L}(V)$, respectively. Refer to [1] for detailed explanations of the equations.

The free and open source software, Scilab (http://www.scilab.org/), was used to numerically solve the above ODEs (initial conditions: $V=-60 \mathrm{mV}, n=0.1, x=0.1, b=0.9$, and $[\mathrm{Ca}]=0.1 \mu \mathrm{M}$ ). The total simulation time was $0.6 \mathrm{~s}$ in all the simulations. System parameters whose values were varied were $I_{a p p}, g_{N a}, g_{\mathrm{Ca}}, g_{K}, g_{K C a}$, and $g_{\mathrm{A}}$. $I_{\text {app }}$ between $0.2 \mathrm{~s}$ and $0.6 \mathrm{~s}$ was varied from $-2.0 \mu \mathrm{A} / \mathrm{cm}^{2}$ to $2.5 \mu \mathrm{A} / \mathrm{cm}^{2}$ at an interval of $0.5 \mu \mathrm{A} / \mathrm{cm}^{2}$, whereas $I_{a p p}$ between $0.0 \mathrm{~s}$ and $0.2 \mathrm{~s}$ was set to zero. Default values of $g_{N a}, g_{C a}, g_{K}, g_{K C a}$, and $g_{A}$ were $20 \mathrm{mS} / \mathrm{cm}^{2}, 1$ $\mathrm{mS} / \mathrm{cm}^{2}, 2 \mathrm{mS} / \mathrm{cm}^{2}, 1 \mathrm{mS} / \mathrm{cm}^{2}$, and $4 \mathrm{mS} / \mathrm{cm}^{2}$, respectively. Each of $g_{\mathrm{Na}}, g_{\mathrm{Ca}}, g_{K}, g_{K C a}$, and $g_{\mathrm{A}}$ was varied to $50 \%$ or $150 \%$ of each default value.

\section{Results}

The previous study had indicated that a hyperpolarizing stimulation of $-3 \mu \mathrm{A} / \mathrm{cm}^{2}$ suppressed spontaneous repetitive spiking [1]. To determine the effect of the hyperpolarizing stimulation on spiking activity in more detail, the present study first investigated the response of the model to hyperpolarizing stimulations of smaller amplitudes under conditions in which all the ionic conductances were set to default values. When a stimulation of $-0.5 \mu \mathrm{A} / \mathrm{cm}^{2}$ was applied to the model, it showed a repetitive spiking behavior, but the frequency of spiking became smaller than that before the stimulation (Figure 1(a)). In contrast, when a stimulation of $-1.0 \mu \mathrm{A} / \mathrm{cm}^{2}$ was applied to the model, it did not show a repetitive spiking behavior, but showed a quiescent state (Figure 1(b)). Based on these results, the present study defined $-1.0 \mu \mathrm{A} / \mathrm{cm}^{2}$ as the stimulation threshold for suppressing repetitive spiking (we hereafter referred to this threshold as the "suppression threshold"). To reveal the relationship between the ionic conductances and the dynamics of the model, the present study next investigated how the suppression threshold changed in response to variations of each ionic conductance.

The effects of variations in the ionic conductance values on the dynamics of the model are shown in Figure 2. Under conditions in which $g_{N a}$ was $50 \%$ of the default value with other conductance values being set at the de- 

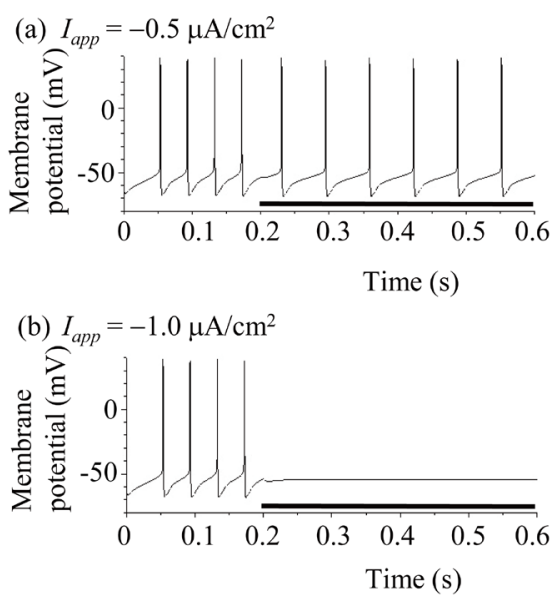

Figure 1. Time courses of the membrane potential of the type-A mVNn model. (a) $I_{a p p}=$ $-0.5 \mu \mathrm{A} / \mathrm{cm}^{2}$. (b) $I_{a p p}=-1.0 \mu \mathrm{A} / \mathrm{cm}^{2}$. Horizontal bars in (a) and (b) indicate the period of the hyperpolarizing stimulation.

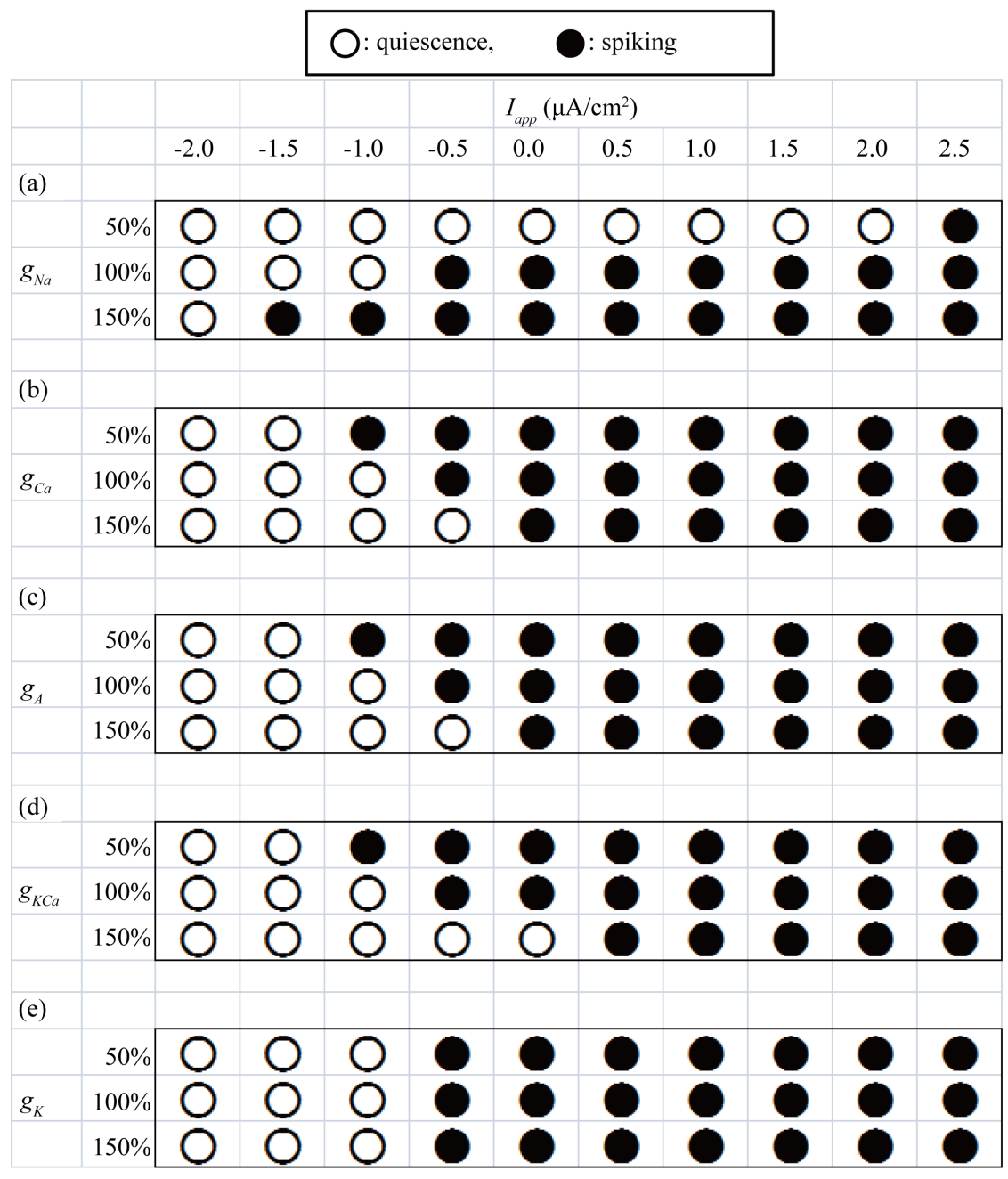

Figure 2. The dependence of the dynamical states of the type-A mVNn model on $I_{a p D}$ and $g_{x}(x=N a, C a, A, K C a$, and $K)$. $\circ$ indicates the quiescent state, whereas $\bullet$ indicates the repetitive spiking state. 
fault values, the model showed a quiescent state in response to stimulation from $-2.0 \mu \mathrm{A} / \mathrm{cm}^{2}$ to $2.0 \mu \mathrm{A} / \mathrm{cm}^{2}$, whereas it showed a repetitive spiking state in response to stimulation of $2.5 \mu \mathrm{A} / \mathrm{cm}^{2}$ (Figure 2(a)). Therefore, the suppression threshold at $50 \% g_{N a}$ was $2.0 \mu \mathrm{A} / \mathrm{cm}^{2}$. Figure 2(a) also shows that the suppression thresholds at $100 \% g_{N a}$ and $150 \% g_{N a}$ were $-1.0 \mu \mathrm{A} / \mathrm{cm}^{2}$ and $-2.0 \mu \mathrm{A} / \mathrm{cm}^{2}$, respectively. Similarly, the suppression thresholds under conditions in which other ionic conductance values were varied were calculated. The suppression threshold at $50 \% g_{C a}, 100 \% g_{C a}$, and $150 \% g_{C a}$ was $-1.5 \mu \mathrm{A} / \mathrm{cm}^{2},-1.0 \mu \mathrm{A} / \mathrm{cm}^{2}$, and $-0.5 \mu \mathrm{A} / \mathrm{cm}^{2}$, respectively (Figure 2(b)). The suppression threshold at $50 \% g_{A}, 100 \% g_{A}$, and $150 \% g_{A}$ was $-1.5 \mu \mathrm{A} / \mathrm{cm}^{2},-1.0 \mu \mathrm{A} / \mathrm{cm}^{2}$, and $-0.5 \mu \mathrm{A} / \mathrm{cm}^{2}$, respectively (Figure 2(c)). The suppression threshold at $50 \% g_{K C a}, 100 \% g_{K C a}$, and $150 \% g_{K C a}$ was $-1.5 \mu \mathrm{A} / \mathrm{cm}^{2},-1.0 \mu \mathrm{A} / \mathrm{cm}^{2}$, and $0.0 \mu \mathrm{A} / \mathrm{cm}^{2}$, respectively (Figure 2(d)). Finally, the suppression threshold at $50 \%, 100 \%$, and $150 \% g_{K}$ was $-1.0 \mu \mathrm{A} / \mathrm{cm}^{2}$ for all three conditions (Figure $2(\mathrm{e})$ ).

\section{Discussion}

The present study performed numerical simulation of the type-A mVNn model and revealed the differential sensitivity of the threshold for the transition from a repetitive spiking state to a quiescent state to variations in the different ionic conductance values. The present study revealed the difference in modulation of the threshold among the ionic conductances. An increase in $g_{N a}$ lowered the threshold, whereas an increase in $g_{C a}, g_{A}$, or $g_{K C a}$ raised the threshold. Finally, an increase in $g_{K}$ had no effect on the threshold. The sensitivity of the threshold to variations in the ionic conductance values decreased in the following order: $g_{N a}>g_{K C a}>g_{C a}=g_{A}>g_{K}$. Although the previous study had investigated the response of the type-A mVNn model to hyperpolarizing and depolarizing stimulation [1], the relationship between the ionic conductances and the threshold for the transition from a repetitive spiking state to a quiescent state had not been clarified prior to the present study.

It is important to determine whether or not variations in system parameters affect mathematical models of neurons linearly. For example, analysis of the ghostbursting model has reported that changes in the dendritic potassium conductance kinetics induce anonlinear effect on the dynamical states in a two-dimensional parameter space of the ghostbursting model [17]. In contrast, in the ghostbursting model, the threshold for the transition from a repetitive spiking state to a bursting state is influenced linearly by several ionic conductances [16]. In addition, one report has indicated that whether or not variations in ionic conductance values affect a certain type of neuron model linearly depends on the type of ionic conductance: in a vibrissa motoneuron model, the threshold for the transition between a subthreshold oscillation state and a tonic firing state changes nonlinearly in response to variations in the M-type potassium and the persistent sodium conductances [15], whereas the threshold for the transition between a subthreshold oscillation state and a mixed-mode oscillation state changes linearly in response to variations in the cationic $\mathrm{h}$ conductance [15]. The study that developed the type-A mVNn model had not investigated the effect of variations in the ionic conductance values on the dynamics of the type-A mVNn model in detail [1]; thus, whether or not variations in the ionic conductance values affect the type-A mVNn model linearly was unclear. The present study resolved this problem: in the type-A mVNn model, the threshold for the transition from a repetitive spiking state to a quiescent state changed linearly in response to variations in $g_{C a}$ and $g_{A}$, whereas the threshold changed highly nonlinearly in response to variations in $g_{N a}$ (the threshold changed slightly nonlinearly in response to variations in $g_{K C a}$ ). In particular, the present study clarified that the dynamics of the type-A mVNn model was affected linearly in response to variations in the calcium conductance. However, because neither the ghostbursting model nor the vibrissa motoneuron model contains the calcium conductance variable [15] [16], whether or not variations in the calcium conductance affects the dynamics of these two models linearly cannot be studied.

\section{Conclusion}

The present study performed numerical simulation of the type-A mVNn model to reveal that the threshold for the transition from a repetitive spiking state to a quiescent state was differentially sensitive to variations in the ionic conductance values among the different types of the ionic conductances, which had not been reported in the previous study of the type-A mVNn model. The present study contributes to a more detailed understanding of the difference among the ionic conductances of the type-A mVNn model.

\section{Acknowledgements}

The author would like to thank Enago (www.enago.jp) for their review of the English language. 


\section{References}

[1] Av-Ron, E. and Vidal, P.P. (1999) Intrinsic Membrane Properties and Dynamics of Medial Vestibular Neurons: A Simulation. Biological Cybernetics, 80, 383-392. http://dx.doi.org/10.1007/s004220050533

[2] Ashrafuzzaman, M. and Tuszynski, J. (2012) Membrane Biophysics. Springer, Heidelberg.

[3] Prinz, A.A., Billimoria, C.P. and Marder, E. (2003) Alternative to Hand-Tuning Conductance-Based Models: Construction and Analysis of Databases of Model Neurons. Journal of Neurophysiology, 90, 3998-4015. http://dx.doi.org/10.1152/jn.00641.2003

[4] Drion, G., Massotte, L., Sepulchre, R. and Seutin, V. (2011) How Modeling Can Reconcile Apparently Discrepant Experimental Results: The Case of Pacemaking in Dopaminergic Neurons. PLoS Computational Biology, 7, Article ID: e1002050. http://dx.doi.org/10.1371/journal.pcbi.1002050

[5] Shirahata, T. (2011) The Effect of Variations in Sodium Conductances on Pacemaking in a Dopaminergic Retinal Neuron Model. Acta Biologica Hungarica, 62, 211-214. http://dx.doi.org/10.1556/ABiol.62.2011.2.11

[6] Soofi, W., Archila, S. and Prinz, A.A. (2012) Co-Variation of Ionic Conductances Supports Phase Maintenance in Stomatogastric Neurons. Journal of Computational Neuroscience, 33, 77-95. http://dx.doi.org/10.1007/s10827-011-0375-3

[7] Vo, T., Bertram, R. and Wechselberger, M. (2013) Multiple Geometric Viewpoints of Mixed Mode Dynamics Associated with Pseudo-Plateau Bursting. SIAM Journal on Applied Dynamical Systems, 12, 789-830. http://dx.doi.org/10.1137/120892842

[8] Shirahata, T. (2014) Effect of Sodium Conductance Variations on Electrical Behavior of a Neocortical Neuron Model. Acta Biologica Hungarica, 65, 379-384. http://dx.doi.org/10.1556/ABiol.65.2014.4.2

[9] Drion, G., O’Leary, T. and Marder, E. (2015) Ion Channel Degeneracy Enables Robust and Tunable Neuronal Firing Rates. Proceedings of National Academy of Sciences, 112, E5361-E5370. http://dx.doi.org/10.1073/pnas.1516400112

[10] Shirahata, T. (2015) Numerical Study of a Mathematical Model of Vibrissa Motoneurons: The Relationship between Repetitive Spiking and Two Types of Sodium Conductance. International Journal of Theoretical and Mathematical Physics, 5, 48-52.

[11] Shirahata, T. (2015) Numerical Simulation Analysis of a Mathematical Model of Circadian Pacemaker Neurons. Applied Mathematics, 6, 1214-1219. http://dx.doi.org/10.4236/am.2015.68113

[12] Shirahata, T. (2016) Quantitative Evaluations of the Contribution of the Excitatory Ionic Conductance to Repetitive Spiking in a Mathematical Model of Medial Vestibular Nucleus Neurons. Acta Biologica Hungarica, 67, 215-219. http://dx.doi.org/10.1556/018.67.2016.2.9

[13] Doiron, B., Laing, C., Longtin, A. and Maler, L. (2002) Ghostbursting: A Novel Neuronal Burst Mechanism. Journal of Computational Neuroscience, 12, 5-25. http://dx.doi.org/10.1023/A:1014921628797

[14] Fernandez, F.R., Mehaffey, W.H. and Turner, R.W. (2005) Dendritic $\mathrm{Na}^{+}$Current Inactivation Can Increase Cell Excitability By Delaying a Somatic Depolarizing Afterpotential. Journal of Neurophysiology, 94, 3836-3848. http://dx.doi.org/10.1152/jn.00653.2005

[15] Golomb, D. (2014) Mechanism and Function of Mixed-Mode Oscillations in Vibrissa Motoneurons. PLoS ONE, 9, Article ID: e109205. http://dx.doi.org/10.1371/journal.pone.0109205

[16] Shirahata, T. (2016) The Relationship of Sodium and Potassium Conductances with Dynamic States of a Mathematical Model of Electrosensory Pyramidal Neurons. Applied Mathematics, 7, 819-823. http://dx.doi.org/10.4236/am.2016.79072

[17] Shirahata, T. (2015) Evaluation of Kinetic Properties of Dendritic Potassium Current in Ghostbursting Model of Electrosensory Neurons. Applied Mathematics, 6, 128-135. http://dx.doi.org/10.4236/am.2015.61013 


\section{Submit or recommend next manuscript to SCIRP and we will provide best service for you:}

Accepting pre-submission inquiries through Email, Facebook, Linkedin, Twitter, etc A wide selection of journals (inclusive of 9 subjects, more than 200 journals)

Providing a 24-hour high-quality service

User-friendly online submission system

Fair and swift peer-review system

Efficient typesetting and proofreading procedure

Display of the result of downloads and visits, as well as the number of cited articles

Maximum dissemination of your research work

Submit your manuscript at: http://papersubmission.scirp.org/ 